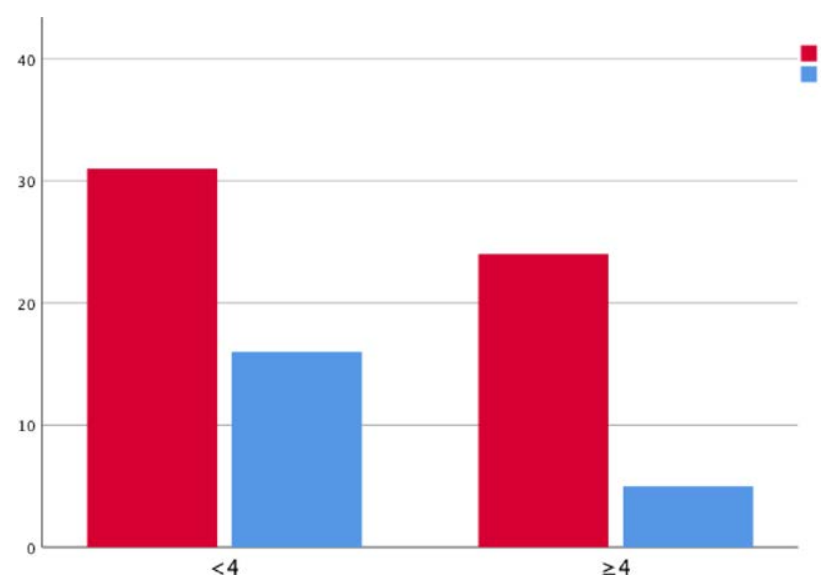

Figure 1. Sarcopenic vs non-sarcopenic patients according to sex

Conclusion: In this study, sarcopenia defined by the SARC-F questionnaire has a predictive value of clinical characteristics of patients to predict sarcopenia parameters and poor physical performance in patients with knee OA. References:

[1] Papalia, R., Zampogna, B., Torre, G., Lanotte, A., Vasta, S., Albo, E., ... \& Denaro, V. (2014). Sarcopenia and its relationship with osteoarthritis: risk factor or direct consequence?. Musculoskeletal surgery, 98(1), 9-14.

Disclosure of Interests: None declared

DOI: 10.1136/annrheumdis-2020-eular.5732

\section{THU0622-HPR PATIENTS' SATISFACTIONS TO TUMOUR NECROSIS FACTOR INHIBITORS FOR MANAGEMENT OF ANKYLOSING SPONDYLITIS IN KOREA; RESULTS FROM A MULTICENTERED, OBSERVATIONAL, AND CROSS-SECTIONAL STUDY}

S. H. Lee', S. G. Lee ${ }^{2}$, Y. G. Kim ${ }^{3}$, S. H. Lee ${ }^{4}$, Y. J. Kim ${ }^{4}$, J.Y. Jeon ${ }^{4}$, H. J. Yoo ${ }^{4}$, J. Lee ${ }^{5}$, T. H. Kim ${ }^{6}$ on behalf of Pfizer Pharmaceuticals Korea Ltd.. ' Kyunghee University, Department of Rheumatology, College of Medicine, Seoul, Korea, Rep. of (South Korea); ${ }^{2}$ Pusan National University Hospital, Pusan National University School of Medicine, Department of Rheumatology, Busan, Korea, Rep. of (South Korea); ${ }^{3}$ University of Ulsan College of Medicine, Asan Medical Center, Division of Rheumatology, Department of Internal Medicine, Seoul, Korea, Rep. of (South Korea); ${ }^{4}$ Pfizer Pharmaceuticals Korea Limited, Division of Medical, Seoul, Korea, Rep. of (South Korea); ${ }^{5}$ Korea University, Department of Biostatistics, College of Medicine, Seoul, Korea, Rep. of (South Korea); ${ }^{6}$ Hanyang University Hospital for Rheumatic Diseases, Department of Rheumatology, Seoul, Korea, Rep. of (South Korea)

Background: An experience of particular treatment is one of the characteristics impacting on patients' decisions to continue, discontinue, or alter their medical treatments. ${ }^{1}$ Reports on patients' treatment satisfaction would be weighty when selecting an optimal treatment reflecting the patient's perspective.

Objectives: We aimed to evaluate the treatment satisfaction of ankylosing spondylitis (AS) patients treated with tumor necrosis factor inhibitors (TNF-Is) and identify the factors associated with satisfaction in Korea.

Methods: The study was conducted from July to November in 2018 at 4 hospitals representative in treating AS in Korea. Patients aged $\geq 19$, diagnosed as AS based on 'Modified New York criteria 1984', and treated with current TNF-Is and same type of device for $\geq 3$ months were included. We used 'Treatment Satisfaction Questionnaire For Medication (TSQM)' which is consisted of 4 domains; effectiveness, side effects, convenience, and global satisfaction. Scores $(0 \sim 100)$ of satisfaction indicate the higher the better. Multivariable linear regressions with $95 \%$ of statistical significance level was performed to identify related factors.

Results: Enrolled 497 patients ( $85.3 \%$ male, 40.3 years old) were prevalent with AS for mean 10 years and indicated average 3.1 in Bath AS Disease Activity Index (BASDAI). Adalimumab (39.6\%) was the most commonly used, followed by etanercept $(23.5 \%)$, infliximab $(21.9 \%)$ and golimumab (14.9\%). TNF-Is were prescribed at low, regular, and high dose for $24.9 \%, 70.5 \%$ and $4.7 \%$ of patients and as subcutaneous with pen type, syringe type, and intravenous injection for $50.9 \%, 26.8 \%$ and $22.3 \%$ of patients, respectively. Conventional disease-modifying antirheumatic drugs(CDMARDs) and steroids were concomitantly used for $5.4 \%$ and $8.3 \%$ of patients, respectively.

Average score of TSQM was the lowest in convenience amongst 4 domains; 72.2 in effectiveness, 96.9 in side effects, 67.6 in convenience, and 71.4 in global satisfaction. Negative associations were shown between BASDAI and all domains of TSQM. Patients who prescribed TNF-Is at high dose were more satisfied to effectiveness while they were less satisfied to side-effect, as against patients prescribed it at low dose. TNF-Is, use of cDMARDs and steroids were not significantly associated with treatment satisfaction (Fig. 1).

Conclusion: AS patients treated with TNF-Is in Korea were quite satisfied with their treatments. Since the worst satisfaction in the convenience domain was presented amongst 4 domains, more convenient treatment options may be required to enhance the entire satisfaction of patients. With the result of a negative association between disease activity and satisfaction, this study strengthened the previous studies reporting the positive relationship between health improvement and satisfaction. ${ }^{2}$

References:

[1] Feighner JP: Impact of anxiety therapy on patients' quality of life. AJM 1987, 82: 14-19.

[2] E. Batbaatar et al. Determinants of patient satisfaction, Perspectives in Public Health (2017) 137(2): 89-101

Figure 1. Related factors with treatment satisfaction measured by TSQM

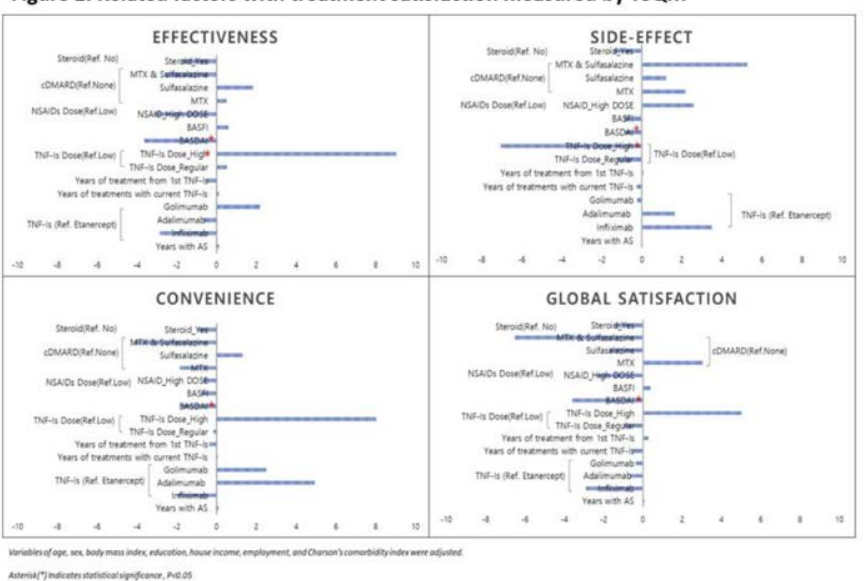

Acknowledgments: Tae-Hwan Kim is the corresponding author.

Disclosure of Interests: Sang-Hoon Lee Grant/research support from: This study was sponsored by Pfizer Pharmaceuticals Korea Limited., Seung Geun Lee Grant/research support from: This study was sponsored by Pfizer Pharmaceuticals Korea Limited., Yong-Gil Kim Grant/research support from: This study was sponsored by Pfizer Pharmaceuticals Korea Limited., Soo-Hyun Lee Employee of: I'm an employee of Pfizer pharmaceuticals Korea, YoungJoo Kim Employee of: I'm an employee of Pfizer pharmaceuticals Korea. Ja-Young Jeon Employee of: I'm an employee of Pfizer pharmaceuticals Korea., Hyung-Jeong Yoo Employee of: I'm an employee of Pfizer pharmaceuticals Korea, Juneyoung Lee Grant/research support from: This study was sponsored by Pfizer Pharmaceuticals Korea Limited., Tae-Hwan Kim: None declared

DOI: 10.1136/annrheumdis-2020-eular.5090

\section{THU0623-HPR REVISION AND VALIDATION OF THE GERMAN VERSION OF THE SYSTEMIC SCLEROSIS QUALITY OF LIFE QUESTIONNAIRE (SSCQOL) WITH MOKKEN SCALE ANALYSIS}

A. Kocher ${ }^{1,2}$, M. Ndosi ${ }^{3}$, K. Hoeper ${ }^{4}$, M. Simon ${ }^{1,2}$, D. Nicca ${ }^{2}{ }^{1}$ Inselspital Bern University Hospital, Bern, Switzerland; ${ }^{2}$ University of Basel, Institute of Nursing Science, Basel, Switzerland; ${ }^{3}$ University of the West of England, Bristol, Department of Nursing and Midwifery, Bristol, United Kingdom; ${ }^{4}$ Hannover Medical School, Department Immunology and Rheumatology, Hannover, Germany

In the validation study, German-speaking SSc patients included in the Swiss MANagement Of Systemic Sclerosis (MANOSS) cross-sectional study completed the revised (polytomous) SScQoL. Mokken model was used to test the construct validity of the scale including its subscales. The scalability $(H$ coefficient) values of $\geq{ }^{0.50}$ are considered "strong," ${ }^{0.49}$ to ${ }^{0.40}$ "moderate" and ${ }^{0.39}$ 